\title{
Engineered biosynthesis of the antiparasitic agent frenolicin $B$ and rationally designed analogs in a heterologous host
}

\author{
Jay T Fitzgerald ${ }^{1}$, Christian P Ridley ${ }^{1,3}$ and Chaitan Khosla ${ }^{1,2}$
}

The polyketide antibiotic frenolicin B harbors a biosynthetically intriguing benzoisochromanequinone core, and has been shown to exhibit promising antiparasitic activity against Eimeria tenella. To facilitate further exploration of its chemistry and biology, we constructed a biosynthetic route to frenolicin B in the heterologous host Streptomyces coelicolor CH999, despite the absence of key enzymes in the identified frenolicin gene cluster. Together with our understanding of the underlying polyketide biosynthetic pathway, this heterologous production system was exploited to produce analogs modified at the C15 position. Both the natural product and these analogs inhibited the growth of Toxoplasma gondii in a manner that reveals sensitivity to the length of the $\mathrm{C} 15$ substituent. The ability to construct a functional biosynthetic pathway, despite a lack of genetic information, illustrates the feasibility of a modular approach to engineering medicinally relevant polyketide products.

The Journal of Antibiotics (2011) 64, 759-762; doi:10.1038/ja.2011.86; published online 21 September 2011

Keywords: antiparasitic antibiotic; biosynthesis; engineering; frenolicin; polyketide

\section{INTRODUCTION}

Type II polyketide synthases (PKSs) catalyze the biosynthesis of the carbon skeletons of numerous polyfunctional aromatic natural products from the actinomycetes, including the clinically important antibiotic oxytetracycline and the anticancer agent doxorubicin. ${ }^{1,2}$ Recent analysis has demonstrated considerable functional modularity within these multienzyme assemblies, which in turn can be exploited to alter priming units and change polyketide backbone length. ${ }^{3,4}$ At the same time, the substrate flexibility of late-stage tailoring enzymes in these natural product pathways can also be exploited to create designer molecules. ${ }^{4,5}$ Here, we have harnessed each of these modular elements to reconstruct a chimeric pathway to the antiparasitic antibiotic frenolicin B (1) $)$, as well as targeted analogs in a heterologous host. Biological evaluation of our analogs against Toxoplasma gondii has revealed the strong sensitivity of this family of antibiotics to the identity of the C-15 substituent, thereby highlighting a new direction for future antiparasitic drug design.

The benzoisochromanequinone (BIQ) antibiotic frenolicin B, produced by the actinomycete Streptomyces roseofulvus, has been extensively investigated as an anticoccidial agent owing to its potent activity against Eimeria tenella. ${ }^{6-9}$ Nonetheless, low fermentation titers $^{10}$ and challenging synthetic procedures ${ }^{11,12}$ appear to have limited further development of this promising anti-infective lead substance. We sought to address both of these problems by reconstructing a biosynthetic pathway to frenolicin B in the heterologous host Streptomyces coelicolor CH999, from which the entire actinorhodin biosynthetic gene cluster has been deleted. ${ }^{13}$

\section{MATERIALS AND METHODS}

General

S. coelicolor $\mathrm{CH} 999 / \mathrm{pBOOST}^{*},{ }^{14}$ which lacks the complete actinorhodin (act) gene cluster, was used as the host for production of polyketides. The plasmid pBOOST $^{*}$ has been shown to cointegrate with vectors containing the SCP2* origin of replication, thereby resulting in higher copy numbers and correspondingly improved antibiotic titers. ${ }^{14}$ The transformation of shuttle vectors bearing BIQ pathway genes into S. coelicolor $\mathrm{CH} 999 / \mathrm{pBOOST}^{*}$ were performed following standard procedure. ${ }^{15}$ Deuterated solvents for NMR experiments were purchased from Cambridge Isotope Laboratories (Andover, MA, USA) and all other solvents were purchased from Fisher Scientific (Pittsburgh, PA, USA) at the highest available grade. ${ }^{1} \mathrm{H}$ and ${ }^{13} \mathrm{C}$ NMR spectra of purified polyketide products were recorded on Varian Inova 500 or $600 \mathrm{MHz}$ instrument (Varian, Walnut Creek, CA, USA) in $\mathrm{CD}_{3} \mathrm{OD}$ or DMSO- $d_{6}$. The ${ }^{1} \mathrm{H}$ NMR spectra were referenced to the solvent peak at 3.31 p.p.m. for $\mathrm{CD}_{3} \mathrm{OD}$ or 2.50 p.p.m. for DMSO- $d_{6}$. The ${ }^{13} \mathrm{C}$ NMR spectra were referenced to solvent at 49.0 p.p.m. for $\mathrm{CD}_{3} \mathrm{OD}$ or 39.52 for DMSO- $d_{6}$. Single-bond ${ }^{1} \mathrm{H}-{ }^{13} \mathrm{C}$, multiple-bond ${ }^{1} \mathrm{H}-{ }^{1} \mathrm{H}$ and ${ }^{1} \mathrm{H}-{ }^{13} \mathrm{C}$ connectivity was determined by HSQC, COSY and HMBC, respectively on Varian Inova-600 NMR instrument. Mass spectra were obtained by ESI at the Vincent Coates Foundation Mass Spectrometry Laboratory at Stanford University.

Construction of shuttle vectors

All cloning steps were performed in either E. coli XL1-Blue (Stratagene, La Jolla, CA, USA) or E. coli DH5 $\alpha$ (MCLab, South San Francisco, CA, USA). Plasmids

\footnotetext{
${ }^{1}$ Department of Chemistry, Stanford University, Stanford, CA, USA and ${ }^{2}$ Department of Chemical Engineering, Stanford University, Stanford, CA, USA 
used for cloning included pUC18 (New England Biolabs, Ipswich, MA, USA) and pET28b (Novagen, now EMD Bioscience, Madison, WI, USA). Expression vectors were constructed using standard molecular biology techniques. See Supplementary Information for a detailed description of the primers and construction.

\section{Production, isolation and characterization of polyketide products} Each transformed strain was grown on R5 agar plates containing $50 \mathrm{mgl}^{-1}$ thiostrepton and $100 \mathrm{mgl}^{-1}$ of apramycin at $30^{\circ} \mathrm{C}$ for 7 days, ${ }^{15}$ after which metabolites were extracted with either $100 \% \mathrm{EtOAc}$ or $\mathrm{EtOAc} / \mathrm{MeOH} /$ acetic acid (89:10:1). This crude material was purified by a variety of methods including preparative HPLC and $\mathrm{NaHCO}_{3}$ extraction. Structural determination was performed using a variety of $2 \mathrm{D}$ heteronuclear NMR experiments and LC/MS. See Supplementary Information for full details.

\section{T. gondii growth inhibition}

Tachyzoites of $T$. gondii strain RH $\Delta$ hxgprt $\mathrm{YFP}^{16}$ were harvested from human foreskin fibroblast (HFF) monolayers grown in $25 \mathrm{~cm}^{2}$ T-flasks. These parasites were then inoculated into a 96-well culture plate containing a confluent layer of HFF cells with $\sim 8 \times 10^{3}$ tachyzoites $/ 0.32-\mathrm{cm}^{2}$ well. Parasites were pre-incubated with $\mathrm{HFF}$ cells for $6 \mathrm{~h}$ at $37^{\circ} \mathrm{C}, 5 \% \mathrm{CO}_{2}$. After pre-incubation, the media was exchanged for fresh DMEM complete ((Dulbecco's modified eagle medium) supplemented with $1 \%$ PS (penicillin-streptomycin) and $10 \%$ fetal bovine serum) containing a given concentration of antiparasitic agent in DMSO. Cultures were then incubated for $72 \mathrm{~h}$ at $37^{\circ} \mathrm{C}$, in $5 \% \mathrm{CO}_{2}$. Parasite growth inhibition was monitored in a SpectraMax Gemini EM fluorescent plate reader (Sunnyvale, CA, USA) at $510 \mathrm{~nm}$ excitation and $540 \mathrm{~nm}$ emission. $\mathrm{ED}_{50} \mathrm{~s}$ were determined using GraphPad Prism (GraphPad Software, La Jolla, CA, USA).

\section{Cytotoxicity assay}

HFF cell cytotoxicity was determined using a MTS (3-(4,5-dimethylthiazol-2yl)-5-(3-carboxylmethoxylphenyl)-2H-tetrazolium) assay (CellTiter 96 Aqueous One Solution Assay; Promega, Madison, WI, USA). HFF cells $\left(2 \times 10^{3}\right.$ cells per well) were grown in a 96-well plate until confluence $(\sim 48 \mathrm{~h})$ in phenol red-free DMEM complete at $37^{\circ} \mathrm{C}, 5 \% \mathrm{CO}_{2}$. Varying concentrations of antiparasitic agent were then added to each well and incubated for $48 \mathrm{~h}$ at which point the medium was replaced with $100 \mu$ l phenol red-free DMEM and $20 \mu \mathrm{l}$ MTS. After an additional $1.5 \mathrm{~h}$ of incubation at $37^{\circ} \mathrm{C}$, and the absorbance at $490 \mathrm{~nm}$ was calculated and the $\mathrm{ED}_{50}$ was determined using GraphPad Prism.

\section{RESULTS AND DISCUSSION}

\section{Determining the tailoring enzymes necessary for BIQ core}

\section{formation}

Before embarking on the reconstruction of the frenolicin pathway, we first attempted to complete the biosynthesis of the simplest BIQ, deoxykalafungin 2a (Scheme 1), ${ }^{17}$ which does not require an initiation module and is an intermediate in the actinorhodin (4) pathway. ${ }^{18}$ Previous studies have shown that introduction of the plasmid pRM5 (which encodes act KS/CLF, ACP, C-9 KR, ARO and CYC genes; for details, see Scheme 1) into $\mathrm{CH} 999$ leads to the production of 3,8dihydroxy-1-methylanthraquinone-2-carboxylic acid (6a) and aloesaponarin II (7a), as summarized in Table $1 .{ }^{13,14}$ The next step in BIQ production is stereoselective reduction of the C-3 ketone into an alcohol capable of cyclizing the third ring of the scaffold (Scheme 1 in red). To accomplish this, we cloned the C-3 ketoreductase gene, encoded by actVI-ORF1, onto pRM5, yielding the plasmid pJF6 (Table 1). As predicted, S. coelicolor $\mathrm{CH} 999 / \mathrm{pJF} 6 / \mathrm{pBOOST}^{*}$ yielded 5a after cyclization and loss of water (Supplementary Figure S16). 5,19

The next step in the pathway is enoyl reduction of $\mathbf{5 a}$ followed by oxygenation. Mutants of the actinorhodin-producing strain $S$. coelicolor A3(2), in which the enoyl reductase gene actVI-ORF2 has been inactivated, produce no actinorhodin, and mutants of the secondary enoyl reductase gene actVI-ORF4 produce only $18 \%$ of the actinorhodin of the native strain. ${ }^{20}$ We therefore constructed two derivatives of pJF6, pCR66 and pCR67, harboring actVI-ORF2 and actVI-ORF2/ actVI-ORF4, respectively (Table 1). The product profiles of $S$. coelicolor

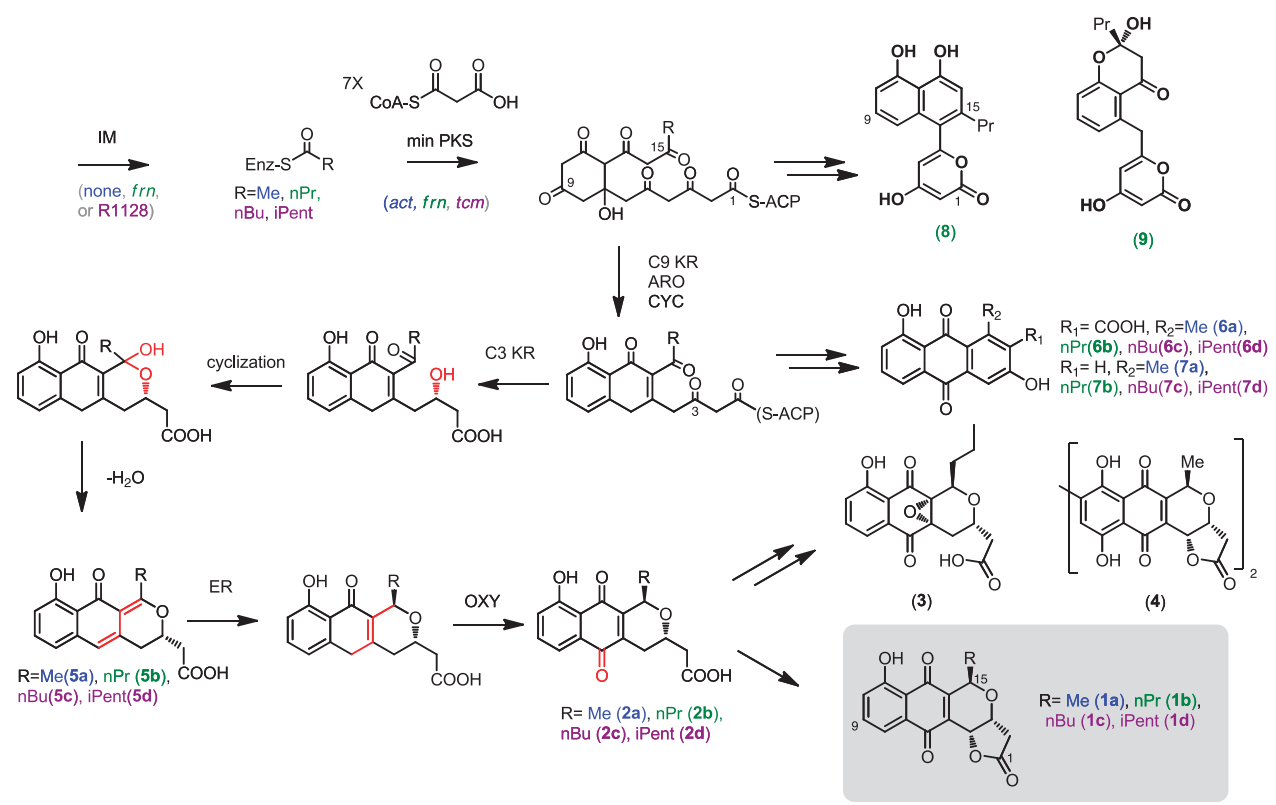

Scheme $1 \mathrm{~A}$ biosynthetic pathway to the benzoisochromanequinones. The presumed biosynthetic route to $1 \mathrm{a}-\mathrm{d}$ is shown along with isolable intermediates and shunt products. The natural product frenolicin A (3) produced by S. roseofulvus, and the natural product actinorhodin (4) produced by S. coelicolor, are also shown as forming from their corresponding carboxylic acids, 2. For details, see text. ARO, 1st ring aromatase encoded by actVII; CYC, 2nd ring cyclase encoded by actIV; C3 KR, C-3 ketoreductase encoded by actVI-ORF1; C9 KR, C-9 ketoreductase encoded by actlII; ER, enoyl reductase encoded by actVIORF2; IM, initiation module (ketosynthase III, acyl carrier protein, acyl-ACP thioesterase); min PKS, minimal polyketide synthase (ketosynthase/chain length factor (KS/CLF), acyl carrier protein (ACP), malonyl-CoA:ACP transacylase (MAT)); OXY, oxygenase, encoded by an unidentified enzyme in the S. coelicolor genome. 
Table 1 Plasmid constructs and resulting polyketide products

\begin{tabular}{|c|c|c|c|c|c|c|}
\hline Plasmida & $K S-C L F$ & $A C P$ & $\begin{array}{l}\text { Initiation } \\
\text { module }\end{array}$ & Other relevant $P K S$ genes & Tailoring ${ }^{b}$ & Polyketide products ${ }^{\complement}$ \\
\hline pRM5 $5^{d}$ & act & act & & act $\mathrm{KR}$, act $\mathrm{ARO}$, act $\mathrm{CYC}$ & actVB & $6 a, 7 a$ \\
\hline pJF6 & act & act & & act $\mathrm{KR}$, act $\mathrm{ARO}$, act $\mathrm{CYC}$ & act $\mathrm{VI-ORF1,} \mathrm{act} \mathrm{VB}$ & $5 a, 6 a, 7 a$ \\
\hline pCR66 & act & act & & act $\mathrm{KR}$, act $\mathrm{ARO}$, act $\mathrm{CYC}$ & actVI-ORF1, actVI-ORF2, actVB & $2 a, 5 a, 6 a, 7 a$ \\
\hline pCR67 & act & act & & act $\mathrm{KR}$, act $\mathrm{ARO}$, act $\mathrm{CYC}$ & act $\mathrm{VI-ORF1,} \mathrm{actVI-ORF2,} \mathrm{act} \mathrm{VI-ORF4,} \mathrm{act} \mathrm{VB}$ & $2 a, 5 a, 6 a, 7 a$ \\
\hline pJF35 & $f r n$ & $f r n$ & $f r n$ & act $\mathrm{KR}$, act $\mathrm{ARO}$, act $\mathrm{CYC}$ & actVI-ORF1, actVI-ORF2, actVB & $2 b, 6 b, 8,9$ \\
\hline pYT127 & tcm & $\mathrm{R} 1128$ & $\mathrm{R} 1128$ & act $\mathrm{KR}$, act $\mathrm{ARO}$, act $\mathrm{CYC}$ & actVB & $6 c, 6 d, 7 c, 7 d$ \\
\hline pJF7 & tcm & R1128 & R1128 & act $\mathrm{KR}$, act $\mathrm{ARO}$, act $\mathrm{CYC}$ & actVI-ORF1, actVB & $5 c, 5 d 6 c, 6 d, 7 c, 7 d$ \\
\hline pJF9 & tcm & $\mathrm{R} 1128$ & $\mathrm{R} 1128$ & act $\mathrm{KR}$, act $\mathrm{ARO}$, act $\mathrm{CYC}$ & act $\mathrm{VI-ORF1,} \mathrm{actVI-ORF2,} \mathrm{actVB}$ & $2 c, 2 d, 5 c, 5 d, 6 c, 6 d, 7 c, 7 d$ \\
\hline
\end{tabular}

aEach pRM5-derived shuttle vector was introduced into S. coelicolor $\mathrm{CH}^{\mathrm{a}} 99 \mathrm{p} / \mathrm{pBOOST}{ }^{*}$ via transformation.

bFunction of gene products: actVB flavin reductase; actVI-ORF1 C-3 ketoreductase; actVI-ORF2 enoyl reductase; actVI-ORF4 secondary enoyl reductase.

'See Supplementary Information for compound characterization.

dPreviously reported constructs (see text).

Table 2 Bioactivity of compounds 1a-d and pyrimethamine

\begin{tabular}{lrcr}
\hline Compound & $\begin{array}{c}\text { T. gondii } \\
E D_{50}(n M)\end{array}$ & $\begin{array}{c}H F F \text { cytotoxicity } \\
E D_{50}(n M)\end{array}$ & $\begin{array}{c}\text { Therapeutic } \\
\text { index }\end{array}$ \\
\hline la & $230 \pm 15$ & $5700 \pm 800$ & 25 \\
lb & $260 \pm 15$ & $2400 \pm 300$ & 9 \\
1c & $420 \pm 30$ & - & - \\
ld & $1200 \pm 60$ & $8000 \pm 2000$ & 7 \\
Pyrimethamine & $820 \pm 60$ & $>50000$ & $>60$
\end{tabular}

Each compound was tested at in least triplicate. The therapeutic index is calculated by dividing the cytotoxic $\mathrm{ED}_{50}$ by the $\mathrm{EC}_{50}$ against $T$. gondii. See Supplementary Figures $\mathrm{S} 1$ and $\mathrm{S} 2$ for growth inhibition curves.

harboring either plasmid was identical, and included $\mathbf{2} \mathbf{a}$ as the next isolable intermediate (Supplementary Figure S16). This finding has two interesting implications for BIQ biosynthesis. First, overexpression of actVI-ORF2 in a pRM5-derived vector obviates the need for a supplementary enoyl reductase in a heterologous system. Second, and more interestingly, the inferred product of the enoyl reductase reaction (Scheme 1 bottom left) was not observed, nor was there a large buildup of 5a. It therefore appears that, although the act gene cluster harbors dedicated oxygenase genes, ${ }^{21,22}$ the $S$. coelicolor genome either has a nonspecific oxygenase capable of catalyzing the same reaction, or that quinone formation proceeds spontaneously in air.

\section{Production of frenolicin B}

Having identified the genes required to produce $2 \mathbf{a}$ in $S$. coelicolor CH999/pBOOST*, we turned our attention to frenolicin B and analogs thereof. Whereas the minimal PKS responsible for frenolicin production has been previously defined, ${ }^{23}$ the initiation module and downstream tailoring enzymes have not yet been characterized. Intriguingly, homologs of the actVI-ORF1 and actVI-ORF2 genes are not known to exist in the frenolicin ( $f r n)$ gene cluster. ${ }^{24}$ In fact, constructs harboring all putative biosynthetic genes from the identified frn gene cluster failed to produce frenolicin or any related BIQ antibiotic (data not shown). In the face of insufficient genetic data, we hypothesized that the tailoring enzymes identified in production of $\mathbf{2 a}$ could be functionally repurposed for the biosynthesis of longer chain analogs. To accommodate the longer alkyl priming chain, we replaced the octaketide act KS-CLF genes with the mixed octaketide/nonaketide frn KS-CLF genes, and also included the genes thought to encode the initiation module of the frn PKS.
The frn gene cluster harbors homologs of the genes encoding the well-characterized initiation module of the R1128 cluster. ${ }^{25}$ Specifically, frnI is a homolog of $z h u \mathrm{H}, f r n \mathrm{~K}$ is a homolog of $z h u \mathrm{C}$ and $f r n \mathrm{~J}$ is a homolog of $z h u \mathrm{~N}$. These genes encode a homodimeric KS responsible for chain initiation, an acyl-ACP thioesterase and a dedicated ACP for the initiation module, respectively. Co-expression of these three frn genes with the genes encoding the frn KS-CLF, the $f r n \mathrm{~N}$ ACP the act C-9 KR, the act ARO and the act CYC, as well as the actVI-ORF1 and actVI-ORF2 genes (pJF35; Table 1) yielded a mixture of butyryl-primed nonaketide derivatives that arise from off-path cyclization reactions (Supplementary Figure S17). In addition, very small quantities of $\mathbf{2} \mathbf{b}$ were also observed. Aside from $\mathbf{6} \mathbf{b}$, the two other major shunt products were propyl analogs of the previously identified compounds dehydromutactin and SEK34. ${ }^{26-28}$ They form either due to the inability of the act ARO to aromatize the first ring or the inability of the act CYC to form the second sixmembered ring (Scheme 1). Notwithstanding the relative abundance of these shunt products, the ability of actVI-ORF1 and actVI-ORF2 to replace the unidentified components of the frenolicin pathway underscores the potential for rational design of a biosynthetic scheme using a diverse PKS toolbox in the face of incomplete genetic information.

\section{Analogs of frenolicin B}

With a route in hand to the bioactive natural product frenolicin $B$, we turned our attention to the rational design of biosynthetic analogs. Earlier work with E. tenella had shown that extension of the C-15 methyl substituent of 1a to a propyl substituent (1b) led to improved antiparasitic activity. ${ }^{6}$ We therefore reasoned that analogs $1 \mathbf{c}$ and $\mathbf{1 d}$ could be useful to investigate the steric effect of the C-15 substituent on biological activity. As chemical synthesis of such analogs is not straightforward, we replaced the genes encoding the frn initiation module and ACP with homologs from the R1128 cluster, which incorporates bulkier primer units into the polyketide backbone. ${ }^{29}$ At the same time, to compensate for the anticipated increase in overall chain length of the polyketide skeleton, we also replaced the frn KSCLF genes with homologs from the decaketide tetracenomycin $(\mathrm{tcm})$ PKS. ${ }^{30}$ In combination with the act $\mathrm{KR}$, act $\mathrm{ARO}$ and act $\mathrm{CYC}$, this hybrid bimodular PKS gene cluster yielded a mixture of compounds, including 6c, 6d, 7c and 7d (pYT127; Table 1). ${ }^{23}$

Further inclusion of the actVI-ORF1 and actVI-ORF2 genes yielded S. coelicolor $\mathrm{CH} 999 / \mathrm{pJF} 9 / \mathrm{pBOOST}{ }^{*}$, which produced a spectrum of products including the desired $\mathbf{2 c}$ and $\mathbf{2 d}$ (Table 1 and Supplementary Figure S18). Although this strain produced a wide range of unchar- 
acterized polyketide products, the ratio of the desired products $2 \mathrm{c}$ and $\mathbf{2 d}$ to the shunt products $\mathbf{6 c}$ and $\mathbf{6 d}$ was considerably higher in this recombinant strain than in S. coelicolor $\mathrm{CH} 999 / \mathrm{pJF} 35 / \mathrm{pBOOST}{ }^{\star}$. The lack of large quantities of butyl- and isopropyl-primed analogs of $\mathbf{8}$ and 9 indicate that act ARO and CYC interface with the $t c m$ min PKS better than with the frn PKS. In addition, this suggests that substrate incompatibility arising from increased alkyl priming unit length is unlikely to be the cause of the large amounts of $\mathbf{8}$ and $\mathbf{9}$ observed in $S$. coelicolor $\mathrm{CH} 999 / \mathrm{pJF35/pBOOST}{ }^{*}$. These rationally designed analogs highlight the versatility of our approach to polyketide construction, and enabled evaluation of their antiparasitic activities.

\section{Biological evaluation against $T$. gondii}

Previous studies with E. tenella showed that the lactone forms of the BIQs $1 \mathbf{a}$ and $\mathbf{1} \mathbf{b}$ were more potent than the free-acid forms ( $2 \mathbf{a}$ and 2b). ${ }^{6}$ We therefore converted our isolated analogs 2 a-d into their corresponding lactones $\mathbf{1 a - d}$ by previously reported methods. ${ }^{31}$ The structures of the novel compounds $\mathbf{1 c}$ and $\mathbf{1 d}$ were confirmed by NMR and mass spectrometric analysis (Supplementary Tables S3, S5 and S6). The compounds were assayed against T. gondii, the causative agent of toxoplasmosis, along with the known antiparasitic agent pyrimethamine as a reference. ${ }^{32}$ A slight modification of a previously reported yellow fluorescence protein reporter assay was used for this purpose $^{16}$ (Supplementary Figures S1 and S2). As summarized in Table 2, within the $\mathrm{C}_{1}-\mathrm{C}_{4}$ range, the alkyl substituent has little effect on antiparasitic activity against T. gondii, but the longer branched substituent leads to a notable decrease in activity. Thus, the benefit of the longer substituent against E. tenella does not hold in our assays, and could be due to improved pharmacokinetics or differential species specificity. To assess their relative tolerability by mammalian cells, we also measured the cytotoxicity of 1a-d against HFF-1 cells using a MTS-based assay (Supplementary Figure S3). The therapeutic indices of 1a-d, reported in Table 2, appear to be comparable.

\section{CONCLUSION}

In summary, notwithstanding incomplete genetic data for the natural product biosynthetic pathway, we have successfully engineered a chimeric, heterologous system for the production of frenolicin B. Furthermore, we have exploited this heterologous system to produce novel frenolicin analogs that are difficult to access by alternative methods. Our results showcase the utility of a modular approach to the creation of medicinally relevant polyketide products of type II PKSs. Last but not least, we have shown that the new compounds have antiparasitic activity against T. gondii in vitro. Further modification of the biosynthetic system presented here could yield additional improvements in BIQ productivity as well as additional analogs of frenolicin B.

\section{ACKNOWLEDGEMENTS}

This research was supported by a grant from the National Institutes of Health to C.K. (R01 CA 77248). T. gondii strain RH 4 xgprt YFP was a gift from Dr Matthew Bogyo. We thank Dr Sandeep Ravindran for his help in culturing T. gondii.

\footnotetext{
1 Khosla, C. \& Ridley, C. P. Encyclopedia of Microbiology 472-481 (Academic Press, San Diego, CA, 2009).
}

2 O'Hagan, D. The Polyketide Metabolites (Ellis Horwood, Chichester, UK, 1991).
3 Das, A. \& Khosla, C. Biosynthesis of aromatic polyketides in bacteria. Acc. Chem. Res. 42, 631-639 (2009).

4 Hertweck, C. The biosynthetic logic of polyketide diversity. Angew. Chem. Int. Ed 48, 4688-4716 (2009).

5 Hopwood, D., Taguchi, T., Ebizuka, Y. \& Ichinose, K. A new mode of stereochemical control revealed by analysis of the biosynthesis of dihydrogranaticin in Streptomyces violaceoruber Tu 22. J. Am. Chem. Soc. 123, 11376-11380 (2001).

6 Omura, S., Tsuzuki, K. \& Iwai, Y. Anticoccidal activity of frenolicin B and its derivatives. J. Antibiot. 38, 1447-1448 (1985).

7 Armer, R. E. et al. Anticoccidial activity of novel semi-synthetic analogues of deoxyfrenolicin and Frenolicin B (Part I). Heterocycl. Commun. 4, 309-315 (1998).

8 Armer, R. E. et al. Anticoccidial activity of novel semi-synthetic analogues of deoxyfrenolicin and Frenolicin B (Part II). Heterocycl. Commun. 4, 345-350 (1998).

9 Armer, R. E. et al. Carbocyclic frenolicin analogues: novel anticoccidial agents. Bioorg. Med. Chem. Lett. 8, 139-142 (1998).

10 Omura, S., Iwai, Y., Awaya, J. \& Oiwa, R. Compound, frenolicin B which is useful as an antibiotic. US Patent 4199514. (1980).

11 Masquelina, T., Hengartnerb, U. \& Streith, J. Naphthopyranquinone antibiotics: novel enantioselective syntheses of frenolicin B and some of its stereoisomers. Helv. Chim. Acta 80, 43-58 (1997).

12 Brimble, M. A., Narin, M. R. \& Prabaharan, H. Synthetic strategies towards pyranonaphthoquinone antibiotics. Tetrahedron 56, 1937-1992 (2000).

13 McDaniel, R., Ebert-Khosla, S., Hopwood, D. \& Khosla, C. Engineered biosynthesis of novel polyketides. Science 262, 1546-1550 (1993).

$14 \mathrm{Hu}$, Z., Hopwood, D. A. \& Hutchinson, C. R. Enhanced heterologous polyketide production in Streptomyces by exploiting plasmid co-integration. J. Ind. Microbiol. Biotechnol. 30, 516-522 (2003).

15 Kieser, T., Bibb, M. J., Buttner, M. J., Chater, K. F. \& Hopwood, D. A. Practical Streptomyces Genetics 472-481 (The John Innes Foundation, Norwich, UK, 2000).

16 Gubbels, M. J., Li, C. \& Striepen, B. High-throughput growth assay for Toxoplasma gondii using yellow fluorescent protein. Antimicrob. Agents Chemother. 47, 309-316 (2003).

17 Hoekesema, H. \& Krueger, W. C. Kalifungin. II. Chemical transformations and the absolute configuration. J. Antibiot. 29, 704-709 (1976).

18 Hopwood, D. A. Genetic contributions to understanding polyketide synthases. Chem. Rev. 97, 2465-2497 (1997).

19 Ichinose, K. et al. Proof that the actVI genetic region of Streptomyces coelicolor A3(2) is involved in stereospecific pyran ring formation in the biosynthesis of actinorhodin. Bioorg. Med. Chem. Lett. 9, 395-400 (1999).

20 Taguchi, T. et al. Chemical characterisation of disruptants of the Streptomyces coelicolor A3 (2) actVI genes involved in actinorhodin biosynthesis. J. Antibiot. 53, 144 (2000).

21 Okamoto, S., Taguchi, T., Ochi, K. \& Ichinose, K. Biosynthesis of actinorhodin and related antibiotics: discovery of alternative routes for quinone formation encoded in the act gene cluster. Chem. Biol. 16, 226-236 (2009).

22 Kendrew, S. G., Hopwood, D. A. \& Marsh, N. G. Identification of a monooxygenase from Streptomyces coelicolor A3(2) involved in biosynthesis of actinorhodin: purification and characterization of the recombinant enzyme. J. Bacteriol. 179, 4305-4310 (1997).

23 Tang, Y., Lee, T. S., Khosla, C. \& Lee, H. Y. Exploring the biosynthetic potential of bimodular aromatic polyketide synthases. Tetrahedron 60, 7659-7671 (2004).

24 Bibb, M. J., Sherman, D. H., Omura, S. \& Hopwood, D. A. Cloning, sequencing and deduced functions of a cluster of Streptomyces genes probably encoding biosynthesis of the polyketide antibiotic frenolicin. Gene 142, 31-39 (1994).

25 Tang, Y., Lee, T. S. \& Khosla, C. Engineered biosynthesis of regioselectively modified aromatic polyketides using bimodular polyketide synthases. PLOS Biol. 2, 227-238 (2004).

26 McDaniel, R., Ebert-Khosla, S., Hopwood, D. A. \& Khosla, C. Engineered biosynthesis of novel polyketides: actVII and actIV genes encode aromatase and cyclase enzymes, respectively. J. Am. Chem. Soc. 116, 10855-10859 (1994).

27 Zhang, H. et al. Mutactin, a novel polyketide from Streptomyces coelicolor. Structure and biosynthetic relationship to actinorhodin. J. Org. Chem. 55, 1682-1684 (1990).

28 Khosla, C. et al. Genetic construction and functional analysis of hybrid polyketide synthases containing heterologous acyl carrier proteins. J. Bacteriol. 175, 2197-2204 (1993).

29 Marti, T., Hu, Z., Pohl, N. L., Shah, A. N. \& Khosla, C. Cloning, nucleotide sequence, and heterologous expression of the biosynthetic gene cluster for R1128, a non-steroidal estrogen receptor antagonist. J. Biol. Chem. 275, 33443-33448 (2000).

30 Bibb, M. J., Biró, S., Motamedi, H., Collins, J. F. \& Hutchinson, C. R. Analysis of the nucleotide sequence of the Streptomyces glaucescens tcml genes provides key information about the enzymology of polyketide antibiotic biosynthesis. EMBO J. 8, 2727-2736 (1989).

$31 \mathrm{Li}, \mathrm{T}$. \& Ellison, R. H. Stereoselective total synthesis of racemic kalafungin and nanaomycin A. J. Am. Chem. Soc. 78, 6264-6265 (1978).

32 McCabe, R. \& Oster, S. Current recommendations and future prospects in the treatment of toxoplasmosis. Drugs 38, 973-987 (1989).

Supplementary Information accompanies the paper on The Journal of Antibiotics website (http://www.nature.com/ja) 